\title{
Meningoencefalites toxoplásmica e chagásica em pacientes com infecção pelo vírus da imunodeficiência humana: diagnóstico diferencial anatomopatológico e tomográfico
}

\author{
Meningoencephalitis due to Toxoplasma gondii and \\ Trypanosoma cruzi in patients with HIV infection. \\ Diferencial diagnosis of pathologic and \\ tomographic findings \\ Javier $\in$. Lazo, Antônio Corlos Oliveirn Meneses, Ademir Rocha, Jack K. Frenkel, \\ Jaime Olavo Marquez, Edmundo Chapadeiro e Edison Reis lopes
}

\begin{abstract}
Resumo Em 22 pacientes com sorologia positiva para o vírus da imunodeficiência humana, com ou sem síndrome da imunodeficiência adquirida, dos quais $7 \mathrm{com}$ meningoencefalite toxoplásmica e 15 com meningoencefalite chagásica associadas, procuraram-se dados diferenciais, entre as duas encefalopatias, tanto à anatomia patológica quanto à tomografia computadorizada do crânio. Os resultados observados e os dados da literatura nos permitiram concluir que enquanto na meningoencefalite necrosante focal por Toxoplasma gondii o acometimento dos núcleos da base é freqüente, na meningoencefalite necrosante focal causada pelo Trypanosoma cruzi, lesões dessas estruturas parecem não ocorrer ou ser excepcionais. De outro lado, o acometimento da substância branca parece nitidamente maior na meningoencefalite chagásica que na meningoencefalite toxoplásmica, ao passo que o parasitismo e a hemorragia do tecido nervoso, bem como as lesões das bainhas de mielina são mais freqüentes e intensos na meningoencefalite causada pelo Trypanosoma cruzi que naquela por Toxoplasma.
\end{abstract}

Palavras-chaves: AIDS. AIDS $x$ doença de Chagas. AIDS $x$ toxoplasmose. Meningoencefalites. Encefalites.

\begin{abstract}
Twenty-two HIV+ patients with encephalitis were studied. Of these, 7 had meningoencephalitis due to Toxoplasma gondii (MT) and 15 due to Trypanosoma cruzi (MC). Pathologic and computerized axial tomography (CAT) changes were compared. We found that focal necrotizing encephalitis due to Toxoplasma involved the cerebral cortex and the basal ganglia, whereas lesions due to Trypanosoma cruzi were centered in the white matter, sometimes extending into the cortex. Hemorrhages, myelin lesions and organisms were more pronounced in chagasic than in toxoplasmic encephalitis. These findings are consistent with the literature reviewed.
\end{abstract}

Key-words: AIDS. AIDS x Chagas' disease. AIDS x toxoplasmosis. Meningoencephalitis. Encephalitis.

Curso de Pós-graduação em Patologia Humana da Faculdade de Medicina do Triângulo Mineiro, Uberaba, MG, Centro de Ciências Biomédicas da Universidade Federal de Uberlândia, Uberlândia, MG, Brasil e Universidade do Novo México, EUA.

Endereço para correspondência: Prof. Edison Reis Lopes. Faculdade de Medicina do Triângulo Mineiro. Av. Getúlio Guaritá 130, 38025440 Uberaba, MG.

Tel: (034) 318-5154. E-mail: pgpathum_fmtm @ mednet.com.br.

Recebido para publicação em 04/08/97. 
Os dados até hoje conhecidos2 9 indicam haver semelhança no comportamento clínico, laboratorial, radiológico e anatomopatológico entre portadores das associações vírus da imunodeficiência humana (HIV) com doença de Chagas (DC) e síndrome da imunodeficiência adquirida (AIDS) com toxoplasmose (T), particularmente nas lesões encefálicas que se desenvolvem em ambas as associações.

$\mathrm{O}$ crescente aumento do número de casos relatados da associação HIV-DC, especialmente no Brasil, na Argentina e no Chile, e a alta freqüência mundial da associação AIDS$T$, levaram-nos a pesquisar dados diferenciais anatomopatológicos e de imagem pela tomografia axial computadorizada do crânio (TAC) entre as encefalopatias chagásica e toxoplásmica, em pacientes $\mathrm{HIV}^{+}$.

\section{MATERIAL E MÉTODOS}

Vinte e dois pacientes com sorologia positiva para HIV, com ou sem AIDS, dos quais 7 apresentaram meningoencefalite toxoplásmica (MT) associada e, 15, meningoencefalite chagásica (MC).

Todos os portadores da associação AIDS-T, bem como dois $\mathrm{HIV}^{+}$com DC estiveram internados, foram submetidos a TAC de crânio e por nós necropsiados. Dos 15 casos de MC, 2 foram, neste estudo, examinados por nós pessoalmente. Os outros 13 casos já haviam sido analisados em trabalho anterior ${ }^{19}$ por alguns de nós. Nenhum dos pacientes com MT recebeu tratamento para esta doença. Nos casos de $\mathrm{MC}$, ou não se empregaram drogas tripanossomicidas ou, quando ministradas, o foram após a obtenção dos dados tomográficos.

Os diagnósticos de AIDS foram baseados nos critérios do "Centers for Disease Control" de Atlanta $^{3}$ e os de MET nos dados clínicos, laboratoriais, tomográficos e pela detecção, ao exame necroscópico, do Toxoplasma gondii. Nos dois pacientes com MC de nossa atual casuística e nos sete com MT, a sorologia para HIV, in vivo, foi positiva pelo método ELISA e confirmada, em quatro, pela reação de imunofluorescência indireta e em um pelo Western blot. Testes de ELISA para HIV, realizados em sangue colhido à necropsia, foram positivos nos 7 pacientes com MT.

Para a análise microscópica os cortes foram corados pela hematoxilina-eosina e, quando necessário, pelo retículo de Wilder, tricrômico de Masson, hematoxilina fosfotúngstica, Verhoeff-Van Gieson e luxol fast blue contracorado com ácido periódico-Schiff (PAS). Para pesquisa e caracterização imuno-histoquímica do $T$. cruzi e do $T$. gondii, utilizou-se a reação da imunoperoxidase indireta, com anticorpos primários policlonais obtidos em coelhos cronicamente infectados na Faculdade de Medicina do Triângulo Mineiro.

\section{RESULTADOS}

Achados anatomopatológicos. Nos chagásicos, os encéfalos apresentavam aumento de peso e volume, alargamento e achatamento dos giros e apagamento dos sulcos. Cortes frontais (Figura 1) mostravam múltiplas áreas amolecidas, hemorrágicas, de limites imprecisos e de tamanho variado, medindo até vários centímetros de diâmetro. As lesões atingiam tanto a substância branca como a cinzenta, parecendo predominar na primeira. Localizavamse nos vários lobos cerebrais, onde eram maiores, ocorrendo também no cerebelo e no tronco cerebral; em nenhum caso havia comprometimento dos gânglios da base. Em particular, nas áreas amolecidas, havia meningoencefalite com necrose (Figuras 5 e 6) e hemorragia. A maioria dos focos necróticos encefálicos apresentavam em sua intimidade hemorragia difusa. O processo inflamatório das leptomeninges tinha intensidade variável, de discreta a moderada, e infiltrado celular constituído especialmente de macrófagos, linfócitos, células plasmáticas e granulócitos neutrófilos e, nem sempre, mantinha relações topográficas com as lesões encefálicas. Nódulos microgliais foram observados, disseminados, nas substâncias branca e cinzenta, em praticamente todos os casos. Edema intracelular, necrose, focos de hemorragia recentes ou mais antigos e gliose focal astrocítica, em focos, ora isolados ora confluentes, localizados especialmente na substância branca, aparentemente sem localização preferencial, foram achados constantes. Também havia denso exsudato de macrófagos, linfócitos, plasmócitos e menor quantidade de neutrófilos nos espaços perivasculares. Freqüentes eram as necroses vasculares mas poucas tromboses foram observadas. Nas áreas necróticas situadas na substância branca, não se identificaram vestígios de mielina nem substâncias coradas, pelo luxol fast blue, nos macrófagos. Na periferia destas áreas necróticas, bem como nas regiões da substância branca, 

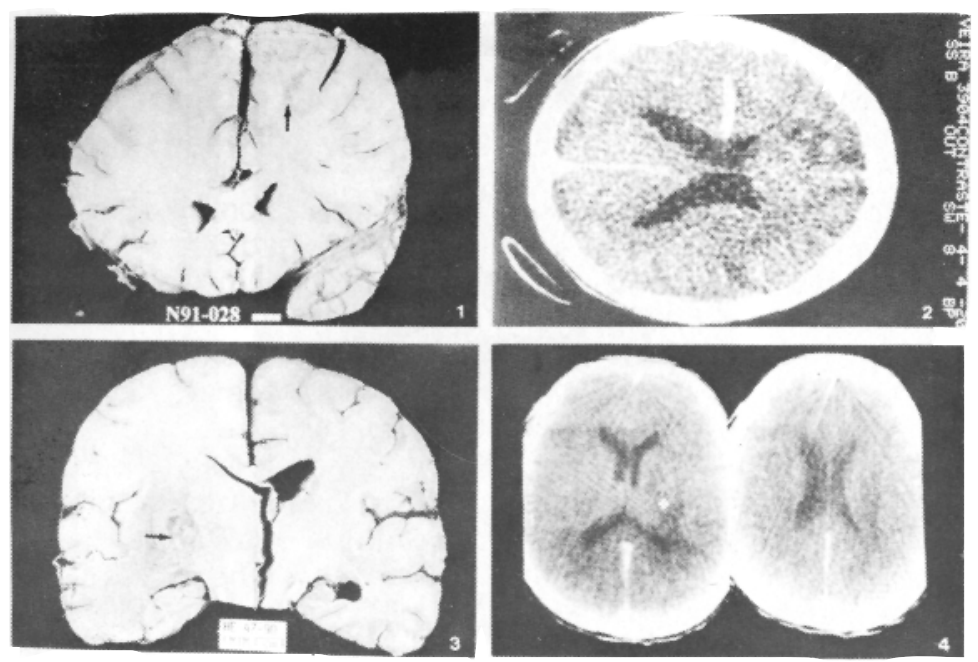

Figura 1 - Meningoencefalite chagásica. Corte frontal. Lesões necrosantes com pontilhados hemorrágicos no giro frontal superior esquerdo (seta).

Figura 2 - Meningoencefalite chagásica. Mesmo caso da Figura 1 em corte horizontal. Tomografia computadorizada do crânio, salientando zona hipodensa (letra $\mathrm{x}$ ).

Figura 3 - Meningoencefalite toxoplásmica. Corte frontal. A seta indica lesão necrosante, no hemisfério cerebral esquerdo, em correspondência com o núcleo lentiforme, cápsula interna e tálamo, estendendo-se ao mesencéfalo (núcleo rubro). Notar desvio da linha média e colapso do ventrículo lateral à esquerda, resultante do efeito de massa produzido pela lesão necrótica.

Figura 4 - Meningoencefalite toxoplásmica. Tomografia computadorizada do encéfalo mostrado na Figura 3. Cortes horizontais. Notar zona hipodensa (asterisco) que corresponde à área necrótica indicada na Figura 3.

havia edema pronunciado; fibras com tumefação e fragmentação da bainha mielinica alternavamse com outras aparentemente íntegras. Havia, em todos os casos, intenso parasitismo de células gliais e talvez macrófagos por formas amastigotas do T. cruzi, especialmente em torno das áreas necro-hemorrágicas (Figura 6) e dos vasos sangüíneos; formas amastigotas isoladas do T. cruzi eram também freqüentes no interstício (Figura 7) e, em um caso, detectaram-se macrófagos parasitados, no espaço subaracnóideo.

Nos 7 pacientes com toxoplasmose, o exame anatomopatológico macroscópico demonstrou encéfalos aumentados de peso e volume, com giros alargados e achatados e apagamento dos sulcos. As leptomeninges eram espessas e turvas. O exame das secções do encéfalo (Figura 3) revelou, em todos os casos, lesões focais, amolecidas, ligeiramente granulosas e friáveis, de tamanho variado, desde poucos milímetros a vários centímetros, por vezes com pontilhados hemorrágicos, sediadas tanto na substância branca como na cinzenta. Nesta, nos 7 casos havia acometimento da córtex e/ou da substância branca subcortical e, em 6 casos $(86 \%)$, as lesões atingiam também os núcleos da base. Microscopicamente, em todos os casos, havia congestão e edema difusos do parênquima encefálico e áreas de necrose por coagulação, de tamanho variados, em diferentes estágios evolutivos, disseminadas nas substâncias branca e cinzenta (Figuras 8 e 9A). Em algumas áreas havia somente tecido necrótico. Em 5 casos, em alguns focos, à necrose associava-se hemorragia discreta com as hemácias dispostas na periferia das lesões e em outros focos, além da necrose associada ou não à hemorragia, constatavam-se infiltrados de macrófagos com restos mielínicos fagocitados, linfócitos e plasmócitos. Nas áreas em que a necrose aparentava ser mais antiga, distinguiam-se halos de edema e hemorragia, os quais pareciam envolver o tecido necrótico. Vasculite era freqüente especialmente na intimidade ou em torno das 


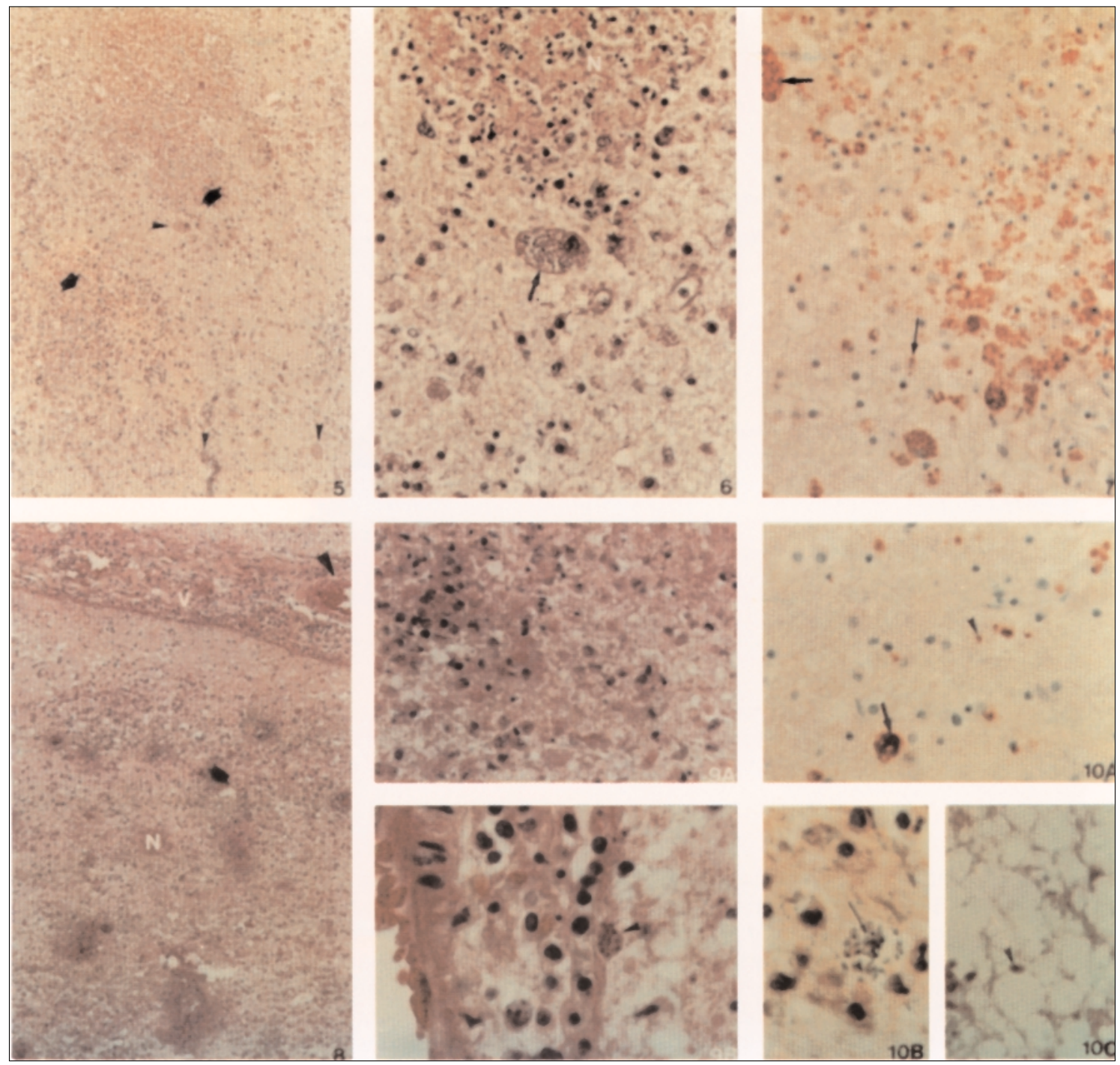

Figura 5 - Meningoencefalite chagásica. Corte de substância branca de cérebro mostrando áreas de necrose (setas) e ninhos de amastigotas do T. cruzi (cabeças de setas). Hematoxilina-eosina 140x.

Figura 6 - Meningoencefalite chagásica. Detalhe da Figura 5 mostrando área de necrose $(\mathrm{N})$ na proximidade da qual observa-se ninho com formas amastigotas do T. cruzi (seta). Hematoxilina-eosina 560x.

Figura 7 - Meningoencefalite chagásica. Corte de área necrótica mostrando intenso parasitismo por formas amastigotas de T. cruzi às quais se apresentam livres no interstício (seta fina) ou formando ninhos (seta larga). Peroxidase indireta e hematoxilina 280x.

Figura 8 - Meningoencefalite toxoplásmica. Corte de encéfalo mostrando área de necrose por coagulação (N) com limite indicado pela seta, rodeada por halo de edema e vasculite (V). Hematoxilina-eosina 140x.

Figura 9 - Meningoencefalite toxoplásmica. A) Detalhe da área de necrose mostrada na Figura 8. Hematoxilina eosina 560x. B) Detalhe da vasculite mostrada na Figura 8, identificando-se taquizoitos de T. gondii formando grupos (cabeça de seta). Hematoxilina-eosina 1000x

Figura 10 - Meningoencefalite toxoplásmica. Cortes de encéfalo onde se identifica T. gondii sob formas livres e formando grupos (cabeças de seta para formas livres e setas para grupos). A) Peroxidase indireta hematoxilina 560x. B) Hematoxilinafosfotúngsticả 560x. C) Hematoxilina-eosina 1000x. 
áreas necróticas (Figuras 8 e 9B). Alguns vasos apresentavam, nos espaços de Virchow-Robin, infiltrados predominantemente de mononucleares; outros mostravam nas paredes exsudato celular, de intensidade variável, formado especialmente por mononucleares. Por vezes, notava-se, ainda, nas paredes vasculares, necrose fibrinóide. Tromboses foram observadas em vasos situados na intimidade das áreas necróticas. A coloração pelo luxol fast blue mostrou, na intimidade das áreas necróticas, fibras com alterações mielínicas, caracterizadas ora por rarefação ora por desorganização da mielina, a qual se apresentava parcialmente destruída ou em arranjos concêntricos grosseiros. Ao lado das fibras lesadas havia outras aparentemente sem alterações morfológicas. Nódulos microgliais, distribuídos por todo o encéfalo, foram vistos em cinco casos. Nos sete casos havia leptomeningite, mais intensa nos locais em que a pia-aracnóide recobria áreas de parênquima necrótico. Nessas regiões, o processo inflamatório se caracterizava por intensa congestão e infiltrado celular constituído predominantemente por mononucleares e com menor número de neutrófilos. Nas demais partes da leptomeninge a inflamação era discreta. Tanto nas colorações rotineiras quanto na imuno-histoquímica e especialmente nesta, com facilidade, identificavam-se formas livres de taquizóitos e cistos de T. gondii (Figuras 10A, B e $C$ ), os primeiros mais freqüentes que os últimos. Na maioria, mas não na totalidade das regiões necróticas, detectaram-se parasitas. Estes também foram vistos próximos aos vasos e nas adventícias e médias dos mesmos. Nos nódulos gliais o parasitismo, quando presente, era discreto. Formas císticas de T. gondii foram observadas também em áreas encefálicas aparentemente sem lesões. Não se observaram parasitas nas meninges.

Achados tomográficos. Os resultados encontram-se nas Tabelas 1 e 2.

Tabela 1 - Achados da tomografia computadorizada do crânio em quinze pacientes com meningoencefalite chagásica.

\begin{tabular}{|c|c|}
\hline Caso & Relatório do exame tomográfico \\
\hline 8 & $\begin{array}{l}\text { Múltiplas lesões nodulares hipodensas em ambos os hemisférios cerebrais, com reforço em forma de anel após injeção do } \\
\text { contraste. }\end{array}$ \\
\hline 9 & Áreas hipodensas nos lobos frontal e parietal direitos (Figura 2). \\
\hline 10 & Hidrocéfalo não comunicante com alargamento dos ventrículos laterais e terceiro ventrículo. \\
\hline 11 & Áreas hipodensas com formações nodulares múltiplas, algumas com reforço em anel e discreto efeito de massa. \\
\hline 12 & $\begin{array}{l}\text { Duas áreas hipodensas, córtico-subcorticais, uma parieto-occipital direita e outra temporo-parietal esquerda, esta menos } \\
\text { definida. Dentro da primeira lesão, formação anular, de } 2 \mathrm{~cm} \text { de diâmetro, com bordas de maior densidade. }\end{array}$ \\
\hline 13 & Lesão irregular, realçada após injeção do contraste, no lobo temporal esquerdo, com edema e efeito de massa. \\
\hline 14 & $\begin{array}{l}\text { Grande lesão hipodensa em lobo frontal direito, com acentuado efeito de massa, colabando a porção frontal do ventrículo } \\
\text { lateral direito e causando significativa herniação subfalcina, reforço digitiforme da lesão hipodensa frontal anterior, após } \\
\text { injeção do contraste. }\end{array}$ \\
\hline 15 & Duas imagens hipodensas à direita, uma no lobo frontal e outra no parietal, com edema e reforço em anel . \\
\hline 16 & Lesão hipodensa, com parede espessada, com reforço em anel, no mesencéfalo. \\
\hline 17 & Múltiplas lesões cerebrais. \\
\hline 18 & $\begin{array}{l}\text { Duas imagens hipodensas nas regiões occipital e parietal esquerdas, de contornos irregulares, sem efeito expansivo, sem } \\
\text { realce após injeção de contraste. }\end{array}$ \\
\hline 19 & Lesões granulomatosas parietal e frontal direitas. \\
\hline 20 & Lesão granulomatosa parietal direita. \\
\hline 21 & Massa fronto-parieto-occipital direita com grande componente edematoso. \\
\hline 22 & $\begin{array}{l}\text { Imagens múltiplas, córtico-subcorticais e vizinhas de gânglios da base, que captam contraste, algumas de forma anular e } \\
\text { com efeito de massa. }\end{array}$ \\
\hline
\end{tabular}

Tabela 2 - Achados da tomografia computadorizada do crânio em três pacientes com meningoencefalite toxoplásmica.

\begin{tabular}{|c|c|}
\hline Caso & Relatório do exame tomográfico \\
\hline 2 & Zona hipodensa na região posterior da cápsula interna à esquerda. Após injeção do contraste não houve realce (Figura 4). \\
\hline 4 & $\begin{array}{l}\text { Zona hipodensa, de } 8 \times 5 \mathrm{~cm} \text {, têmporo-parietal direita, com acentuado efeito de massa sem reforço significativo após } \\
\text { administração de contraste. Desvio do III ventrículo e colabamento do ventrículo lateral direito. }\end{array}$ \\
\hline 5 & $\begin{array}{l}\text { Lesões hipodensas nas regiões frontal, parietal e talâmica à direita e na região occipital à direita e à esquerda sem reforço } \\
\text { significativo após administração do contraste. Dilatação ventricular. }\end{array}$ \\
\hline
\end{tabular}




\section{DISCUSSÃO}

Tanto na reativação da $\mathrm{DC} 17$ quanto na da toxoplasmose 1 podem se desenvolver meningoencefalites com características necrosantes similares, embora seja possível estabelecer-se entre elas algumas diferenças morfológicas.

Parece existir diferença macroscópica quanto à sede das lesões. Estas, nas duas encefalopatias, se caracterizam por focos inflamatórios necrosantes, hemorrágicos ou não, múltiplos ou únicos, localizando-se tanto na substância branca quanto na cinzenta. Na MC, parecem predominar na substância branca e, na MT, na cinzenta. Nesta última, os núcleos da base são mais acometidos que na MC, segundo se pode deduzir, pelo cotejo de nossos dados com os da literatura. Post e cols 16 , Chimelli e cols 4 e Chimelli e Rosemberg5, com base em dados anatomopatológicos, afirmam que tálamo e/ou núcleos da base são, na MT, áreas preferencialmente acometidas pelas lesões necrosantes e, segundo Macedo10, as lesões na MT têm predileção pelos núcleos da base e pela união do córtex e substância branca subcortical. Em todos os nossos 7 aidéticos com MT, o estudo anatomopatológico mostrou que as lesões acometeram a substância cinzenta e/ou a substância branca subcortical, e em $6(86 \%)$, atingiram também os núcleos da base. Contrariamente, nos dois casos de MC que analisamos, as lesões localizavam-se, predominantemente, na substância branca e em nenhum havia comprometimento dos núcleos da base. Os dados da literatura, também aqui, parecem corroborar nossos achados. Os trabalhos 713141920 , que descrevem os achados macroscópicos na MC de imunodeprimidos, não fazem referência a lesões dos núcleos da base e, da leitura destes estudos, fica a impressão de que o acometimento da substância branca pelas lesões necrosantes é mais comum que o da cinzenta. Não temos hipoteses para explicar a predileção, na toxoplasmose, pelas lesões dos núcleos da base.

Microscopicamente, é também possível detectar-se alguns dados diferenciais entre as duas associações em análise. Os casos, aqui estudados, deixaram a impressão de que, tanto na meningoencefalite por toxoplasma quanto naquela causada pelo T. cruzi, as lesões básicas se localizavam inicialmente na substância branca. Com o evoluir do processo, parecem ter se estendido à substância cinzenta, o que, a nosso ver, sucede mais na MT que na DC. É provável que esta seja a razão de se observar, mais comumente na MT que na chagásica, lesões das substâncias branca subcortical e cinzenta. Os achados histopatológicos confirmam a observação macroscópica previamente relatada.

Embora nas áreas de necrose de ambas as encefalopatias, tenham sido detectados focos de hemorragia, estes, eram difusos na $\mathrm{MC}$ e focais e periféricos na MT. Em 20 (87\%) dos 23 portadores da associação Chagas e AIDS, estudados por Rocha e cols ${ }^{19}$, havia hemorragia associada à necrose. Chimelli e cols 4 também chamam a atenção para a ocorrência de hemorragia nas lesões nodulares necróticas da MC. Alguns autores, por nós já referidos, por vezes assinalam a ocorrência de focos de hemorragia na MT sem, entretanto, enfatizá-los. Em nossa casuística, hemorragia encefálica foi observada nos dois chagásicos e em cinco dos sete toxoplasmóticos. Além disto, em ambos os chagásicos havia, também, focos de hemorragia em anel não detectados em nenhum dos nossos casos de MT.

Diverso, também, o comportamento do parasitismo nas meningoencefalites chagásica e toxoplasmótica. Tanto nos preparados corados pela hematoxilina-eosina quanto naqueles com reações imuno-histoquímicas demonstram muitos T. cruzi e relativamente poucos T. gondii. Este achado parece de importância na análise histopatológica de fragmentos obtidos através de biópsias estereotáxicas, técnica já algumas vezes empregada no diagnóstico da MC7 13.

Um quarto aspecto microscópico que nos parece, até certo ponto, diverso refere-se a alterações da mielina. Estas nos pareceram mais intensas nos casos de meningoencefalites chagásicas que nas toxoplasmóticas. Ainda que estes achados morfológicos devam ser interpretados com cautela, visto que não é possível determinar se o tempo de evolução das lesões nas duas encefalopatias é similar, cremos ser possível levantar a hipótese de que as alterações das fibras mielínicas são mais intensas na MC que na MT.

Em quinto lugar, nos parece merecedor de discussão o diferente comportamento das leptomeninges nas duas encefalopatias. Os dados conhecidos e os do presente estudo 
sugerem que o acometimento inflamatório da leptomeninge parece mais intenso e difuso na MC do que na MT. T. gondii não foi identificado nas leptomeninges nos casos por nós estudados. Embora trabalhos 61121 façam referência à presença destes protozoários no líquido cefalorraquidiano (LCR), pelo que pudemos averiguar, nenhum dos estudos anatomopatológicos 121516 relatados na literatura não mencionam o achado de T. gondii em preparados histopatológicos de leptomeninge de portadores de MT. O encontro de T. cruzi, pelo que nos consta, somente ocorreu em um caso de MC19. Contrariamente ao que se relata na MT, o achado de T. cruzi no LCR em chagásicos imunossuprimidos com MC parece freqüente2 1119.

Finalmente, uma referência sobre as lesões vasculares nas duas encefalopatias em discussão. Vasculites necrosantes e tromboses foram por nós observadas e têm sido descritas por Bertoli e cols 1 e Huang e Chou8, na encefalite toxoplásmica, e por Rocha e cols ${ }^{19}$ e Oddo e cols ${ }^{14}$, na MC. Os trabalhos sobre MT são mais numerosos, abrangem casuística mais amplas e tratam da questão em maior profundidade do que na MC. Fundamentados na nossa experiência e nos dados da literatura, temos a impressão de que as lesões vasculares necrosantes e as tromboses são mais freqüentes e intensas na MT do que na MC, o que poderia explicar o encontro de focos de necrose sem parasitas, no casos de MT. Entretanto, julgamos que há necessidade de mais estudos, em especial na MC, para se ter uma idéia definitiva sobre $o$ assunto.

Quanto à TAC, segundo os nossos achados e os dados da literatura2 1215161819 , nas duas

Tabela 3 - Principais características diferenciais entre meningoencefalites necrosantes focais por T. cruzi e por T. gondii.

\begin{tabular}{|c|c|c|}
\hline & \multicolumn{2}{|c|}{ Meningoencefalite necrosante focal por } \\
\hline & Trypanosoma cruzi & Toxoplasma gondii \\
\hline \multirow[t]{2}{*}{ Sede das lesões } & $\begin{array}{l}\text { Mais na substância branca do que } \\
\text { na cinzenta. }\end{array}$ & $\begin{array}{l}\text { Predominam na substância cinzenta e branca } \\
\text { subcortical }\end{array}$ \\
\hline & Não descritas nos núcleos da base & Freqüentes nos núcleos da base \\
\hline Hemorragia nas áreas necróticas & Difusa & Periférica \\
\hline Parasitas em tecidos & Abundantes & Menos numerosos \\
\hline Lesões mielínicas & Freqüentes e de maior intensidade & Poucas e de menor intensidade \\
\hline Leptomeningite & Difusa de intensidade variável & $\begin{array}{l}\text { Focal em correspondência com as lesões } \\
\text { parenquimatosas }\end{array}$ \\
\hline Vasculites necrosantes e tromboses & Menor freqüência e intensidade & Maior freqüência e intensidade \\
\hline
\end{tabular}

encefalopatias em discussão, ocorrem lesões simples ou múltiplas, uni ou bilaterais, redondas, iso ou hipodensas, com ou sem reforço em anel após contraste, que podem ou não apresentar efeito de massa. O exame dos nossos casos mostra que em 6 dos 7 chagásicos submetidos a contraste ocorreu reforço em anel, achado não detectado nos 3 pacientes com toxoplasmose. A leitura dos trabalhos de Navia e cols12, Porter, Sande 15 , Renold e cols 18 e Post e cols 16 indica que em até $85 \%$ dos portadores de MT a TAC pode revelar reforço em anel das lesões. Nossa pequena casuística não nos permite analisar a razão da discordância destes achados. Quanto à sede das lesões, os achados tomográficos de Navia e cols 12 , Porter, Sande 15 , Renold e cols 18 e Wanke e cols22 indicam que os núcleos da base são freqüentemente acometidos na MT. Em oposição, em nenhum caso de MC, detectaram-se lesões destes núcleos. $O$ trabalho de Roland e Loyola20 refere lesão chagásica vizinha dos núcleos da base, mas não menciona alterações na intimidade destas estruturas.

A Tabela 3 sintetiza os principais dados diferenciais entre as duas encefalopatias por

\section{AGRADECIMENTOS}

nós abordadas.

Ao Conselho Nacional de Desenvolvimento
Científico e Tecnológico (CNPq) e à Fundação de Amparo à Pesquisa do Estado de Minas 


\section{REFERÊNCIAS BIBLIOGRÁFICAS}

Gerais (FAPEMIG) pelo apoio financeiro.

1. Bertoli F, Espino M, Arosemena JR, Fishback JL, Frenkel JK. A spectrum in the pathology of toxoplasmosis in patients with acquired immunodeficiency syndrome. Archives of Pathology and Laboratory Medicine 119:214-224, 1995.

2. Burgarelli MKN. Contribuição para o estudo clínicolaboratorial e imunopatológico da associação entre doença de Chagas e a síndrome da imunodeficiência adquirida. Revista de Patologia Tropical 25:81-167, 1996.

3. Centers for Disease Control. Revision of the CDC surveillance case definition for acquired immunodeficiency syndrome. Morbidity and Mortality Weekly Report 36(supl 1):1S-15S, 1987.

4. Chimelli L, Basílio de Oliveira CA, Torres W, Torres MC, Miyagui T, Meneses DMF, Valle HA. Patologia da AIDS. In: Brasileiro Filho G, Pittella JEH, Pereira FEL, Bambirra EA, Barbosa AJA (eds) Bogliolo patologia. 5 ${ }^{\underline{a}}$ edição. Guanabara Koogan, Rio de Janeiro, p.1087-1102, 1994.

5. Chimelli L, Rosemberg S. Neuropatologia da síndrome da imunodeficiência adquirida (AIDS). Revista da Sociedade Brasileira de Medicina Tropical 26:113-119, 1993

6. Dement SH, Cox MD, Gupta PK. Diagnosis of central nervous system Toxoplasma gondii from the cerebrospinal fluid in a patient with acquired immunodeficiency syndrome. Diagnostic Cytopathology 3:148-151, 1987.

7. Gluckstein D, Ciferri F, Ruskin J. Chagas' disease: another cause of cerebral mass in the acquired immunodeficiency syndrome. American Journal of Medicine 92:429-432, 1992.

8. Huang TE, Chou SM. Oclusive hypertrophic arteritis as the cause of discrete necrosis in CNS toxoplasmosis in the acquired immunodeficiency syndrome. Human Pathology 19:1210-1214, 1988.

9. Lazo JE. Diagnóstico diferencial entre meningoencefalites toxoplásmica e chagásica em pacientes com infecção pelo vírus da imunodeficiência humana. Ênfase às alterações morfológicas. Tese de Mestrado. Faculdade de Medicina do Triângulo Mineiro, Uberaba, MG, 1995.

10. Macedo V. Toxoplasma. In: Castro LP, Cunha AS, Rezende JM (eds) Protozooses humanas. Fundo Editorial BYK, São Paulo, p. 153-170, 1994.

11. Medeiros EAS, Roland H, Loyola S. Acute
Trypanosoma cruzi meningoencephalitis in patients with AIDS. In: Resumos da 32nd Interscience Conference on Antimicrobial Agents and Chemotherapy, Anaheim, p. 173, 1992.

12. Navia BA, Petito CK, Gold JWM, Cho E, Jordan BD, Price RW. Cerebral toxoplamosis complicating the acquired immune deficiency syndrome: clinical and neuropathological findings in 27 patients. Annals of Neurology 19:224-238, 1986.

13. Nunes Neto A, Medeiros EA, Neto MC, Stavale JN, Júnior GT, Pereira CAP. Meningoencefalite chagásica em AIDS: relato de três casos. Revista da Sociedade Brasileira de Medicina Tropical 24(supl 1):23, 1991.

14. Oddo D, Casanova M, Acuña G, Ballesteros J, Morales B. Acute Chagas' disease (trypanosomiasis americana) in acquired immuno-deficiency syndrome: report of two cases. Human Pathology 23: 41-44, 1992.

15. Porter SB, Sande MA. Toxoplasmosis of the central nervous system in the acquired immunodeficiency syndrome. New England Journal of Medicine 327:1643-1648, 1992.

16. Post DMJ, Chan JC, Hensley GT, Hoffman TA, Moskowitz LB, Lippmann S. Toxoplasma encephalitis in Haitian adults with acquired immunodeficiency syndrome: a clinical-pathologic-CT correlation. American Journal of Roentgenology 140:861-868, 1983.

17. Prata A. Chagas' disease. Infectious Diseases Clinics of North American 8:61-76, 1994.

18. Renold C, Sugar A, Chave JP, Perrin L, Delavelle J, Pizzolato G, Burkhard P, Gabriel V, Hirschel B. Toxoplasma encephalitis in patients with the acquired immunodeficiency syndrome. Medicine 71:224-239, 1992.

19. Rocha A, Meneses ACO, Silva AM, Ferreira MS, Nishioka SA, Burgarelli MKN, Almeida E, Turcato G, Metze K, Lopes ER. Pathology of patients with Chagas' disease and acquired immunodeficiency syndrome. The American Journal of Tropical Medicine and Hygiene 50:261-266, 1994.

20. Roland M, Loyola, S. Reactivación atípica de la enfermedad de Chagas en un paciente con SIDA. In: Resumos das 18 ${ }^{\text {a }}$ Jornadas Nacionales Interresidencias de Clínicas Médica, Buenos Aires, p. 113. 1991.

21. Vietzke WM, Gelderman AH, Grimley DM, Valsamis MP. Toxoplasmosis complicating malignancy: Experience at the National Cancer Institute. Cancer 21:816-827, 1968. 
22. Wanke C, Tuazon CU, Kovacs A, Dina T, Davis DO, Barton N, Katz D, Lunde M, Levy C, Conley FK, Lane $\mathrm{HC}$, Fauci AS, Masur H. Toxoplasma encephalitis in patients with acquired immune deficiency syndrome: diagnosis and response to therapy. The American Journal of Tropical Medicine and Hygiene 36:509- 